\title{
Correction to: Inhibitor discovery from pomegranate rind for targeting human salivary a-amylase
}

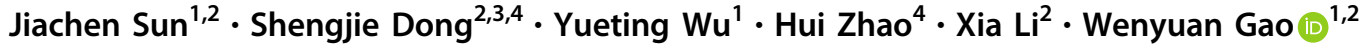

Published online: 28 June 2018

(c) Springer Science+Business Media, LLC, part of Springer Nature 2018

Correction to: Medicinal Chemistry Research (2018) 27:1559-1577 https://doi.org/10.1007/s00044-018-2164-2.

The original version of this article unfortunately contained error in the captions of Figs. 10-12. The correct figure captions are presented with this erratum.

Fig. 10 Hydrophobic surface calculation of a rutin, b luteolin, c quercetin, and d kaempferol with HAS plotted using Chimera. In Chimera, amino acid residues are automatically assigned an attribute named kdHydrophobicity, with values according to the hydrophobicity scale of Kyte and Doolittle

Fig. 11 Electrostatic surface calculation of $\mathbf{a}$ rutin, $\mathbf{b}$ luteolin, c quercetin, and $\mathbf{d}$ kaempferol with HSA plotted using Chimera. Coulombic surface coloring calculates electrostatic potential according to Coulomb's law: $\varphi=\Sigma$ $\left[q_{\mathrm{i}} /\left(\varepsilon d_{\mathrm{i}}\right)\right]$, where $\varphi$ is the potential (which varies in space), $q$ are the atomic partial charges, $d$ are the distances from the atoms, and $\varepsilon$ is the dielectric constant, representing screening by the medium or solvent. A distance-dependent dielectric ( $\varepsilon=C \mathrm{~d}$, where $C$ is some constant) is sometimes used to approximate screening by implicit solvent

Fig. 12 Coulombic potential mapping on the molecular surface of a rutin, $\mathbf{b}$ luteolin, $\mathbf{c}$ quercetin, and $\mathbf{d}$ kaempferol in terms of quantum chemical calculations

The original article can be found online at https://doi.org/10.1007/ s00044-018-2164-2.

$\triangle \mathrm{Xia} \mathrm{Li}$

lixia2008@tju.edu.cn

$\triangle$ Wenyuan Gao

pharmgao@tju.edu.cn

1 Tianjin University of Traditional Chinese Medicine, 300193 Tianjin, China

2 School of Pharmaceutical Science and Technology, Tianjin University, 300072 Tianjin, China

3 Faculty of General Education, Guangdong Baiyun University, 510450 Guangzhou, China

4 Department of Physics, Tianjin Normal University, 300387 Tianjin, China 